\title{
MESSAGE FROM THE ORGANIZING COMMITTEE
}

Welcome to the Brazilian Breast Cancer Symposium 2018.

Dreams are meant to come true. Goiania Breast Cancer Symposium (GBCS) has been constantly growing during its seven editions. Thus, it was necessary to broaden new horizons, think big and envision the growth of the entire breast cancer scientific community. Therefore, in order to be regarded as an actual international event, and with the concept that all Brazilian regions could be represented, the GBCS name has been changed to BBCS - Brazilian Breast Cancer Symposium. We believe that this change will bring the awareness to researchers, participants and sponsors that this is a continental event, with a real possibility to grow even further.

On the other hand, such growth could not lead to the loss of the intimate nature that belongs to the culture of those who live in the Middle-West of Brazil and the expectations regarding the formation of new research groups, associations and businesses. Hence, the charming and picturesque city of Pirenópolis is a suitable place to host the event. Pousada dos Pirineus is a cozy place keeping the charm and the intimate atmosphere we longed for, but above all it can inspire the participants to jointly search new ideas, and especially new solutions related to the diagnosis, treatment and rehabilitation of breast cancer.

Another important change was the publication of abstracts in a specialized journal. The synergy between the Brazilian Society of Mastology and the Organizing Committee of the BBCS enabled this extra edition of mastology. Thus, each abstract can be reached easily, allowing citation and making it perennial.

Welcome everyone and make the most of every moment, every idea, every friendship and all the learning you can acquire during this time in Pirenópolis.

Ruffo Freitas-Junior

Rosemar Macedo Sousa Rahal Régis Resende Paulinelli 